\title{
Parietal Influence on Temporal Encoding Indexed by Simultaneous Transcranial Magnetic Stimulation and Electroencephalography
}

\author{
Martin Wiener, ${ }^{1}$ Dasha Kliot, ${ }^{1}$ Peter E. Turkeltaub, ${ }^{2}$ Roy H. Hamilton, ${ }^{1}$ David A. Wolk, ${ }^{1}$ and H. Branch Coslett ${ }^{1}$ \\ ${ }^{1}$ Department of Neurology, Perelman School of Medicine of the University of Pennsylvania, Philadelphia, Pennsylvania 19104, and ${ }^{2}$ Department of \\ Neurology, Georgetown University School of Medicine, Washington, DC 20007
}

Previous studies have suggested that contingent negative variation (CNV), as recorded by electroencaphalography (EEG), may serve as an index of temporal encoding. The interpretation of these studies is complicated by the fact that, in a majority of studies, the CNV signal was obtained at a time when subjects were not only registering stimulus duration but also making decisions and preparing to act. Previously, we demonstrated that repetitive transcranial magnetic stimulation (rTMS) of the right supramarginal gyrus (rSMG) in humans lengthened the perceived duration of a visual stimulus (Wiener et al., 2010a), suggesting the rSMG is involved in basic encoding processes. Here, we report a replication of this effect with simultaneous EEG recordings during the encoding of stimulus duration. Stimulation of the rSMG led to an increase in perceived duration and the amplitude of $\mathrm{N} 1$ and $\mathrm{CNV}$ components recorded from frontocentral sites. Furthermore, the size of the CNV amplitude, but not N1, positively correlated with the size of the rTMS effect but negatively correlated with bias (the baseline tendency to report a comparison stimulus as shorter), suggesting that the $\mathrm{CNV}$ indexes stimulus duration. These results suggest that a feedforward mechanism from parietal to prefrontal regions mediates temporal encoding and demonstrate a dissociation between early and late phases of encoding processes.

\section{Introduction}

Temporal expectation, the ability to predict the onset of an upcoming stimulus, is intrinsically bound to the perception of temporal duration. Humans possess a remarkable ability to both react to upcoming stimuli and perceive duration across a wide range of timescales (Buhusi and Meck, 2005); however, the biological underpinnings of this ability have yet to be fully elucidated. Both expectation and temporal duration may be indexed by contingent negative variation (CNV), a slow cortical potential characterized by a developing negative polarity at frontocentral scalp locations (Walter et al., 1964; Macar and Vidal, 2004; Fan et al., 2007). The CNV signal develops while a subject is either explicitly or implicitly encoding stimulus duration (Macar and Vidal, 2004; Praamstra et al., 2006) or expecting the temporally predictable onset of a cued imperative stimulus (Scheibe et al., 2010).

Numerous studies have investigated the role of the CNV in explicit temporal processing (Macar and Vidal, 2004; Xuan et al., 2009; Tarantino et al., 2010). A number of these studies suggest that CNV amplitude indexes a temporal accumulation process,

Received May 24, 2012; revised July 2, 2012; accepted July 11, 2012.

Author contributions: M.W. and D.A.W. designed research; M.W., D.K., P.E.T., R.H.H., and H.B.C. performed research; D.A.W. and H.B.C. contributed unpublished reagents/analytic tools; M.W. analyzed data; M.W. wrote the paper.

This project was supported by National Institute of Mental Health Grant MH076227 (H.B.C.).

Correspondence should be addressed to Dr. Martin Wiener, Department of Neurology, University of Pennsylvania, 3720 Walnut Street, Philadelphia, PA 19104-6241. E-mail: wimartin@psych.upenn.edu.

DOI:10.1523/JNEUROSCI.2511-12.2012

Copyright $\odot 2012$ the authors $\quad 0270-6474 / 12 / 3212258-10 \$ 15.00 / 0$ with higher amplitudes associated with subjectively longer intervals (Macar et al., 1999; but see Kononowicz and van Rijn, 2011). However, the majority of these studies have focused on activity during temporal comparison during which the subject must compare the currently elapsing duration with a memory representation and prepare a motor response. As such, CNV activity during this time period may reflect multiple processes (Ikeda et al., 1997). The interpretation of CNV activity is further confounded by evidence suggesting an involvement in expectancy and preparatory processes (van Rijn et al., 2011).

Recently, we (Wiener et al., 2010a) demonstrated that repetitive transcranial magnetic stimulation (rTMS) of the right supramarginal gyrus (rSMG) selectively increased the perceived duration of a visual stimulus during temporal discrimination. The increase in perceived length did not depend on a motor response and occurred with stimulation during both the standard and comparison intervals, suggesting an involvement in temporal encoding processes. Although our results demonstrated that the rSMG stimulation modulates encoding of stimulus duration, they do not reveal possible downstream effects at distal neural sites.

In the present study, we further examined the effect of rTMS stimulation of the rSMG using simultaneous electroencephalographic (EEG) recordings. Subjects received stimulation before the onset of the standard stimulus, when temporal encoding is engaged. This allowed us to investigate the effects on subsequent components free from motor preparation and decision processes. We reasoned that, if the rSMG and the medial frontal structures that generate the $\mathrm{CNV}$ are part of the neural circuitry 


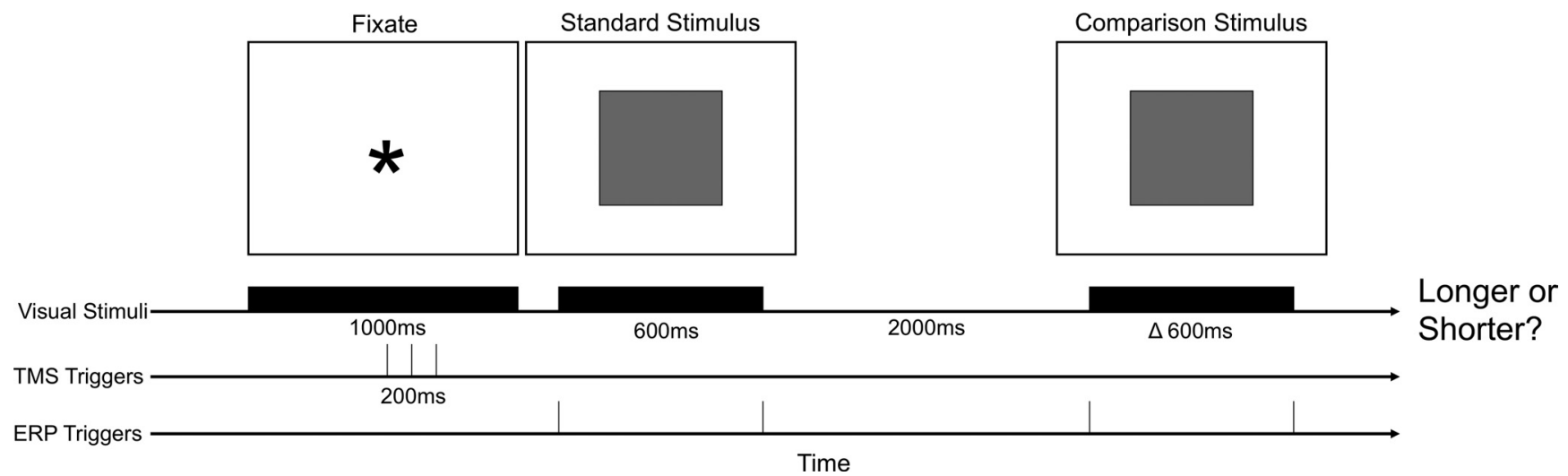

Figure 1. Schematic of the experimental design. Subjects viewed a fixation point for $1000 \mathrm{~ms}$; on TMS trials, subjects received a $200 \mathrm{~ms}$ burst of $10 \mathrm{~Hz}$ stimulation. After the fixation point, a red square standard stimulus was presented for $600 \mathrm{~ms}$. After a $2000 \mathrm{~ms} \mathrm{ISI}$, subjects viewed a comparison stimulus either $600 \mathrm{~ms}$ or a longer or shorter duration individually determined by the DL of baseline responses. Event-related potential (ERP) triggers were coincident with the onset of the standard and comparison stimuli. Subjects were required to indicate with a key press whether they judged the comparison stimulus as longer or shorter than the standard stimulus.

that encodes temporal duration, stimulation of the rSMG would lead to a prolongation of perceived duration, and this increase would be accompanied by a larger CNV amplitude during interval encoding. In contrast, if the CNV indexes other factors, such as memory or decision-making (Macar and Vidal, 2003; Kononowicz and van Rijn, 2011), one would not expect rTMS to induce a change in CNV amplitude during temporal encoding.

\section{Materials and Methods}

Participants. Nineteen right-handed healthy volunteers (10 males, 9 females; mean age, 25 years; range, $21-35$ years) who met inclusion criteria for rTMS participated in the experiment. All subjects gave their informed consent as approved by the University of Pennsylvania Institutional Review Board.

Baseline scaling. To maximize the effects of rTMS on behavior, we individually scaled task difficulty according to level of performance (Wiener et al., 2010a). Before receiving rTMS, each subject performed a baseline temporal discrimination task. Temporal discrimination requires subjects to judge whether a comparison duration is longer or shorter than a standard duration presented on every trial. In this baseline testing, we used the parameter estimation by sequential testing (PEST) algorithm (Pentland, 1980) that provides a continuously updated maximum-likelihood estimate of the threshold for detecting a difference between the comparison and standard durations (Lieberman and Pentland, 1983). With this procedure, the range and frequency of comparison stimuli are different for each subject, thus allowing task difficulty to be scaled to individual performance. We set the initial lower (LT) and upper (UT) thresholds to equal 50 and $150 \%$ of the standard interval duration, respectively. At the onset of each trial, subjects were presented with a fixation point for $1000 \mathrm{~ms}$, followed by the presentation of a red square $(4 \times 4 \mathrm{~cm})$ for $600 \mathrm{~ms}$ (standard duration). After the target interval was reached, the red square extinguished for a $1 \mathrm{~s}$ interstimulus interval (ISI). A second red square was then presented for a variable duration of time (comparison duration) as determined by the maximum-likelihood procedure of the PEST algorithm. The initial stepsize for adjustments in the comparison duration was set to $15 \%$ of the standard duration for the first 20 trials and then to $5 \%$ for the remaining 40 trials. Subjects were required to indicate on a keyboard whether the second stimulus was shorter or longer than the first stimulus; the key mappings used for longer and shorter were counterbalanced between subjects. Once a response was detected, the next trial began immediately. All subjects were given 30 practice trials with a standard interval of $1000 \mathrm{~ms}$ and then completed 60 trials of the baseline task with the $600 \mathrm{~ms}$ standard duration. Subjects were not told the range of stimulus durations and were not given feedback regarding accuracy. Stimulus presentation and the PEST algorithm were performed using MATLAB (MathWorks) with the Psychophysics Toolbox extensions (Brainard, 1997).
The probability of the subject making a "longer" response choice was plotted as a function of the comparison interval (for a demonstration of the technique, see Wiener et al., 2010a). These data were then fit with a sigmoidal, psychometric curve using the psignifit version 2.5.6 software package (see http://bootstrap-software.org/psignifit/) for MATLAB, which implements the maximum-likelihood method described by Wichmann and Hill (2001a). UTs and LTs, the approximate points at which the subject is 25 or $75 \%$ likely to judge the stimulus as longer, were calculated using the bias-corrected bootstrap method implemented by psignifit, based on 4999 simulations (Wichmann and Hill, 2001b). The results of this analysis yield the point of subjective equality (PSE; the time value at which subjects were equally likely to judge the stimulus as longer or shorter) and the difference limen [DL; the difference between the UT (75\%) and LT (25\%) values]. A third measure derived from this procedure is the constant error term (CE; PSE - standard duration); this measure was included to index the subjective bias a subject may have in baseline responses. The CE provides an index of time-order error, with negative values indicating a bias to respond "longer" and positive values a bias to respond "shorter" (Hellström, 1985).

Task design. For the stimulation task, subjects performed a temporal discrimination task similar to the one used during baseline testing (Fig. 1). At the onset of each trial, subjects viewed a fixation point for $1000 \mathrm{~ms}$; on stimulation trials, subjects received a brief train of rTMS $500 \mathrm{~ms}$ into the fixation point. The stimulation parameters differ here from our previous study (Wiener et al., 2010a) in that, in our previous study, rTMS stimulation was concurrent with visual stimulus onset. We chose to stimulate earlier to remove any confounding effects from the TMS pulse on EEG recordings. The effect of a brief train of rTMS stimulation is suggested to last on the order of $1 \mathrm{~s}$ beyond the train (Berardelli et al., 1998); as such, we expected the effect of rTMS to persist during the standard stimulus presentation. The fixation point was followed by the presentation of a red square $(4 \times 4 \mathrm{~cm})$ for $600 \mathrm{~ms}$. After a $2 \mathrm{~s} \mathrm{ISI}$, a second red square was presented for the comparison duration. After the comparison stimulus duration, subjects made a response using the same keys as during the baseline, which initiated the next trial; no response window was used. To scale performance, three durations were chosen for the comparison duration set for each subject: $600 \mathrm{~ms}$ and the individually determined UT and LT values for responding longer on the PEST temporal discrimination task. This procedure was used by centering the DL derived for each subject over the standard duration (mean UT $=694 \pm 40$ $\mathrm{ms}$; mean LT $=511 \pm 34 \mathrm{~ms}$ ). After the second square extinguished, subjects were again required to indicate on a keyboard whether the second stimulus was shorter or longer than the first stimulus; the same key mappings for longer and shorter as in the baseline testing were used for each subject. A single session consisted of 180 trials, with 60 trials for each trial type. Subjects received rTMS stimulation on half of the trials in a given session (90 trials) with 30 trials for each presentation condition. 
Subjects participated in two separate sessions on 2 different days with different stimulation sites on each day.

rTMS stimulation. Stimulation was administered with a Magstim Rapid magnetic stimulator connected to a figure-of-eight air-cooled coil with a diameter of $70 \mathrm{~mm}$. Motor thresholds (MTs) were collected for each subject by determining the stimulation output that elicited a motor response from the contralateral hand on at least $50 \%$ of pulses. The EEG cap introduced a small gap $(7 \mathrm{~mm})$ between the coil and scalp surface; as such, all MT measures were taken after the cap and electrodes were applied. Stimulation remained at this output for the remainder of the experiment.

We used Brainsight Stereotaxic Frameless Software (Rogue Research) to target brain sites for stimulation. A high-resolution MPRAGE image was obtained for each subject and used to construct a three-dimensional image of the subject's brain. A Polaris optical tracking system (Northern Digital) was used with Brainsight to coregister the subject's brain and coil. The anterior aspect of the rSMG, as well as a site in the midline occipital (Mid-Occ)-parietal junction, was identified for each subject (average Talairach coordinates of rSMG: 55, $-30,40$; Mid-Occ-parietal junction: $0,-74$, 48; Fig. 2); the midline site was chosen to serve as a control site for nonspecific effects of rTMS. The location on the EEG cap of stimulation was approximately over CP6 and Pz for rSMG and Mid-Occ-parietal junction stimulation, respectively; this choice of electrode sites for stimulation of our cerebral anatomic regions of interest corresponds to recent work exploring cranio-cerebral correlates (Koessler et al., 2009). Subjects were stimulated at $100 \%$ of their MT with three pulses of rTMS at $10 \mathrm{~Hz}$ ( $\sim 50 \mu$ s pulse, $100 \mathrm{~ms}$ interpulse interval). Stimulation was elicited with a custom script, written in E-Prime (Psychology Software Tools). Subjects sat with their heads in a chin rest to restrict movement. All subjects wore earplugs to reduce noise from coil stimulation.

Behavioral analysis. Subject performance was assessed by examining the proportion of trials on which subjects responded "longer" at each comparison duration on both TMS and non-TMS trials. A $2 \times 2 \times 3$ repeated-measures ANOVA with brain region (rSMG, Mid-Occ), stimulation (TMS, non-TMS), and duration (LT, $600 \mathrm{~ms}$, UT) as withinsubjects factors was conducted. Post hoc tests of specific effects were evaluated with paired $t$ tests. A covariate for CE was also included to control for the effects of response bias; although all subjects confirmed that they understood the instructions and performed a practice version of the task before baseline, responses biases could still exist in the data. For example, subjects could be biased to respond "shorter" across all duration lengths. Because the effect of rTMS was hypothesized to induce a shift in the perceived duration of the standard interval, this effect may be confounded by bias. We therefore included the bias covariate to measure the response to rTMS independent of bias.

EEG recording. Continuous EEG data were recorded by a highimpedance BioSemi Active-Two system with a 32-channel montage of Ag/ $\mathrm{AgCl}$ active electrodes embedded in an elastic cap according to the 10-20 international system. An additional montage of four facial EMG electrodes was used to aid in the detection in eye blinks and saccades. Data were recorded with a sampling rate of $512 \mathrm{~Hz}$. Electrode offset was kept between -20 and $+20 \mu \mathrm{V}$ during all recording sessions. The BioSemi Active-Two system has been used previously for TMS-EEG (LevitBinnun et al., 2010) and was designed to accommodate the effects of rTMS with a high dynamic range, preventing the electrodes from becoming saturated by the magnetic artifact generated by the TMS pulse. To minimize the scalp-to-coil distance, the electrode(s) underneath the coil were removed as necessary during both MT estimation and experimental sessions; missing electrodes were spline interpolated to provide full coverage of all 32 electrodes (Murray et al., 2008). All subjects were instructed to avoid blinking during the experimental session; subjects were allowed to blink during the response period after the comparison stimulus presentation. Visual inspection of the data after recordings verified that subjects understood this instruction, because majority of blinks occurred after the comparison duration or during the ISI.

EEG analyses. After data acquisition, continuous data for each subject were offline bandpass filtered between 30 and $0.01 \mathrm{~Hz}$ and referenced to the common average of all available channels. Channels with excessive noise or artifacts that could not be interpolated were removed from additional analyses $(n=11)$. Ocular artifacts resulting from eyeblinks were removed by designing a spatial filter for artifact components (Pfleiger, 2001; www.sourcesignal.com/papers.html), implemented in EMSE (Source Signal). A similar technique was used for removing artifacts resulting from rTMS stimulation. The artifact elicited by the TMS pulse is characterized by a high-amplitude event with an exponential return to baseline, lasting on the order of 5-20 ms (Walsh and PascualLeone, 2003; Veniero et al., 2009). Although our temporal window of interest was beyond the alleged reach of artifact saturation, we sought to minimize the impact on EEG analysis. We designed spatial filters for each subject by using a method for temporally constrained independent component analysis (ICA). ICA has been used in EEG research for eye-blink artifact removal (Jung et al., 2000) and has been used previously for the removal of TMS artifacts (Iwahashi et al., 2009; Hamidi et al., 2010; Korhonen et al., 2011; for a similar approach using principal component analysis, see Levit-Binnun et al., 2010). Temporally constrained ICA was conducted by visual inspection of the continuous EEG data to identify a subset of the data containing only the artifact and a second subset with clean data. The corresponding spatial filter $(F)$ used for artifact removal has the form: $F=C^{+1 / 2}\left(I-U_{r} U_{r}^{T}\right) C^{-1 / 2}$, where $C$ represents the $32 \times$ 32 covariance matrix for all electrode channels identified in the subset of clean data, and $U$ represents the artifact subspace. $C^{+1 / 2}$ and $C^{-1 / 2}$ represent the respective symmetric and inverse symmetric square root of $C$, obtained by singular value decomposition. As such, the inverse symmet- 
a

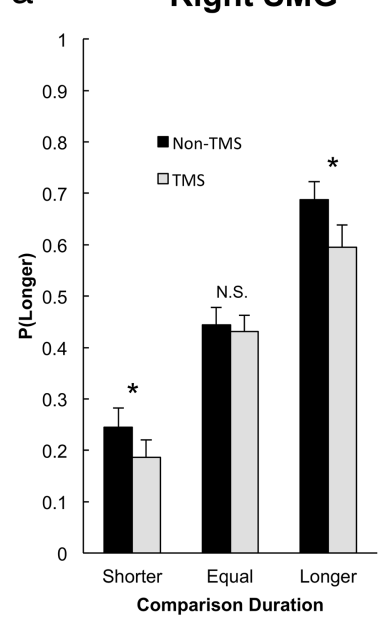

Mid Occ

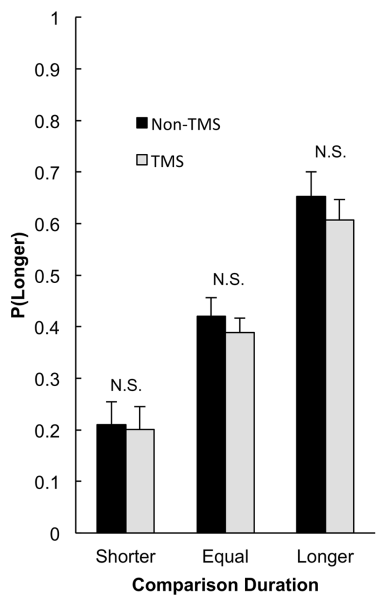

b
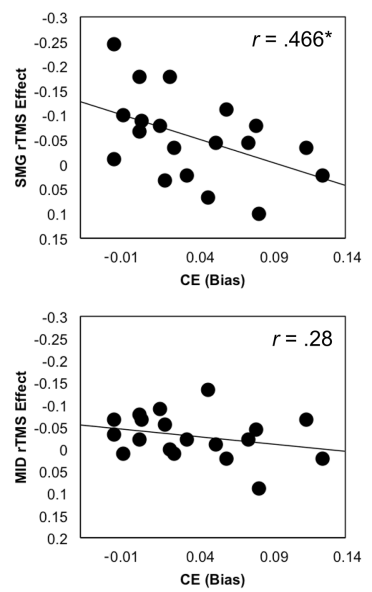

Figure 3. Behavioral effect of stimulation on temporal discrimination. $\boldsymbol{a}$, Subjects exhibited a significant decrease in the proportion of trials on which they judged the second stimulus as longer on rTMS trials $\left({ }^{*} p<0.05\right)$. $\boldsymbol{b}$, Correlation coefficients between $C E$, derived from baseline procedures indicating bias, and mean behavioral effect; larger CE scores indicate an inherent bias to judge comparison stimuli as shorter. rTMS effects were calculated as the mean difference in responding "Ionger" across duration lengths. Subjects with larger positive CE scores were less likely to see an effect of rSMG stimulation.

ric square root spatially whitens the data by the clean subset, whereas the symmetric square roots spatially unwhitens the output. The result thus accentuates artifact-containing data while attenuating clean EEG data. Because of the large-amplitude difference between artifact and clean datasets, components resulting from TMS were easily distinguishable from those with clean data. Components resulting from the TMS artifact were identified and spatially filtered from the continuous data, reducing both the TMS artifact and resulting high-amplitude noise (for a demonstration of the technique, see Iwahashi et al., 2009).

After artifact correction, continuous data were separately epoched with respect to the onset of the standard and comparison stimuli $(-100$ $\mathrm{ms}$ prestimulus onset to $1000 \mathrm{~ms}$ poststimulus onset). All epochs were baseline and DC corrected to the mean of the $100 \mathrm{~ms}$ prestimulus interval. Trials with voltage $\pm 90 \mu \mathrm{V}$ from baseline were discarded. To interrogate the $\mathrm{CNV}$ signal, grand-averaged waveforms across subjects were constructed for frontocentral electrode sites (Fz, Cz, FC1, FC2), in which the CNV signal is reported to be maximal (Macar and Vidal, 2004; Brunia et al., 2011). To identify measurement windows, we used global field power (GFP; Lehmann and Skrandies, 1980), calculated as the root mean square of the amplitude difference across all electrodes. GFP thus provides a polarity and reference-free measure of the electrical field (Murray et al., 2008). Using the GFP from the non-TMS trial data for both stimulation sites, we identified two distinct peaks of GFP activity. We thus divided our analyses between "early" (50-180 ms) and "late" (280-740 $\mathrm{ms}$ ) EEG responses. When projected back onto the referenced data, the early time window encompassed the N1 visual onset response, whereas the late time window encompassed the CNV. This technique has been used previously to identify the CNV time window during time perception (Ng et al., 2011); furthermore, we note that the onset of the late time window corresponds to those used by previous studies of the CNV during standard duration encoding (Gontier et al., 2007; Le Dantec et al., 2007; Chen et al., 2010).

Correlational analyses. To evaluate the effect of rTMS at the individual level, we conducted correlational analyses between the behavioral effects of rTMS and changes in CNV amplitude. For behavioral analyses, we calculated the mean difference in the proportion of longer trials, collapsed across duration length $\left[P(L)_{\text {non-TMS }}-P(L)_{\text {TMS }}\right]$. For EEG analyses, we calculated the mean difference in EEG amplitude for early and late measurement windows ( $\left.\mathrm{EEG}_{\mathrm{TMS}}-\mathrm{EEG}_{\text {non-TMS }}\right)$.

\section{Results}

\section{Behavioral results}

Similar to our previous results (Wiener et al., 2010a), rTMS produced a prolongation of the perceived duration of the standard stimulus (Fig. 3a). A repeatedmeasures ANOVA revealed a significant effect of stimulation $\left(F_{(1,17)}=21.594, p=\right.$ $0.0002)$, characterized by a reduction in the proportion of trials on which subjects judged the second stimulus as longer. Post hoc tests demonstrated that rTMS decreased the proportion of longer responses for the comparison stimulus in the shorter $\left(t_{(18)}=-2.534, p=0.021\right)$ and longer $\left(t_{(18)}=-2.816, p=0.011\right)$ for the rSMG stimulation condition only, with no other effects being significant (all $p>0.05$ ); however, we note that we did not detect an overall interaction between stimulation and brain region $\left(F_{(1,17)}=\right.$ $2.681, p>0.05)$. This is likely attributable to a lack of effect in the equal condition when the standard and comparison stimuli were the same duration. We detected no main effect of brain region $\left(F_{(1,17)}=\right.$ 0.757, $p>0.05)$ or interaction with duration length $\left(F_{(2,34)}=\right.$ $0.761, p>0.05)$. Additionally, we discovered a significant interaction with stimulation and our bias covariate $\left(F_{(1,17)}=6.262\right.$, $p=0.023$ ). Post hoc tests demonstrated a continuous effect of bias; larger baseline constant-error values (indicating positive time-order error and an inherent bias to respond "shorter" for comparison stimuli) were associated with a smaller effect of stimulation. This was manifested as a positive correlation between the mean difference in responding "longer" for rTMS and CE (Pearson's $r=0.466, p=0.044)$; notably, this effect was only found for rSMG and not Mid-Occ stimulation $(r=0.28, p>0.05)$ (Fig. $3 b)$. Positive time-order errors in the present study may relate to adaptation-level effects resulting from the use of the same visual stimulus for both standard and comparison or to the inclusion of a fixation point before standard stimulus onset. In addition, we tested for a relationship between baseline bias and variance. Previous studies suggest that, when sensory evidence is noisier, subjects rely more on internal estimates that are more subject to bias (Jazayeri and Shadlen, 2010) and may increase variability (Cicchini et al., 2012). We compared CE values with our measure of variance (DL) derived from baseline testing; no correlation between $\mathrm{CE}$ and DL values was found $(r=-0.15, p>0.05)$. Additionally, a linear regression test on the effect of CE and DL on the behavioral data found no mediating effect of variance on the either $\operatorname{rSMG}\left(t_{(18)}=-0.299, p>0.05\right)$ or Mid-Occ $\left(t_{(18)}=\right.$ $-0.572, p>0.05)$ stimulation. This finding confirms the independence of bias and variance on timing behavior (Cicchini et al., 2012) and further suggests that the behavioral effect of TMS was not influenced by baseline variability.

To rule out the effect of response hand, a separate ANOVA was conducted with the hand for responding "longer" as a between-subject factor. No main effect of hand $\left(F_{(1,17)}=0.156\right.$, $p>0.05)$ was detected or any interaction with experimental variables (all $p>0.05$ ). Also, to rule out a difference in responding between days, we conducted a separate analysis on the non-TMS trials alone; no significant differences between day of stimulation 
(first or second) was found $\left(F_{(1,17)}=1.961, p=0.179\right)$ or interaction with duration $\left(F_{(1,17)}=0.755, p>0.05\right)$.

We also tested for the effect of rTMS click trains on the behavioral responses. Previous reports have demonstrated that a series of rapid auditory clicks can induce longer estimations of perceived intervals (Penton-Voak et al., 1996). The discharge of the rTMS unit in our paradigm elicited a click for each pulse, which may have influenced the observed effect; indeed, in our previous report, stimulation of the vertex induced a shift similar to but smaller than right rSMG stimulation. Previous investigations on the effect of click trains demonstrated that the loudness of the clicks increases the size of the perceptual shift, suggesting an impact on arousal mechanisms (Burle and Casini, 2001). In the present study, the level of TMS stimulation was scaled to the individual MT; higher MTs are associated with louder clicks during rTMS. Therefore, the effect of click trains constituted a natural experiment within our study that would permit us to explore the effect of click loudness. When MT was included as a covariate, an interaction with the effect of rTMS was found only for Mid$\operatorname{Occ}\left(F_{(1,17)}=8.988, p=0.008\right)$ and not $\operatorname{rSMG}\left(F_{(1,17)}=0.07, p>\right.$ $0.05)$ stimulation. The effect for control site stimulation was also continuous, with higher MT values associated with larger shifts in reporting the second stimulus as shorter (Pearson's $r=-0.588, p=0.008)$. This finding suggests that the effect of click trains did influence the behavioral effect of rTMS but that the effect was restricted to control site stimulation. Furthermore, this finding demonstrates that, in addition to task difficulty, individual differences in the impact of click trains on timing ability were also scaled between subjects. Notably, when we included both the bias and MT covariates in our original analysis, thus controlling for the influence of each parameter, we detected a significant brain region by stimulation interaction $\left(F_{(1,16)}=4.553, p=0.049\right)$, suggesting that the effect of rSMG stimulation on behavior was not attributable to the influence of subject bias and click trains.

\section{EEG results}

We examined both standard and comparison stimulus interval waveforms (Fig. 4a). Consistent with previous reports, grandaveraged waveforms constructed for both visual stimuli exhibited sustained negative potentials that were maximal at frontocentral sites (Fig. $4 b$ ). To examine the effect of rTMS on CNV amplitude, we first ruled out any effect of rTMS on the $\mathrm{CNV}$ waveforms during the comparison stimulus interval, for which we predicted no effect of rTMS. A repeated-measures ANOVA with Greenhouse-Geiser correction on the mean amplitude measures collapsed across frontocentral sites revealed no main effect of brain region $\left(F_{(1,18)}=0.109, p>0.05\right)$, stimulation $\left(F_{(1,18)}=0.0005, p>0.05\right)$, or interaction $\left(F_{(1,18)}=0.025, p>0.05\right)$, indicating that rTMS effects did not extend into the comparison stimulus duration. A significant main effect of measurement window was detected, with the $\mathrm{CNV}$ portion exhibiting higher negative amplitudes than the early N1 portion $\left(F_{(1,18)}=29.803, p=0.00002\right)$, consistent with previous data (Chen et al., 2010). No other interactions were significant (all $p>0.05$ ). A second analysis demonstrated no difference in either measurement window in non-TMS conditions between the days of stimulation (all $p>0.05$ ).

For the standard stimulus duration, we did not detect a main effect of brain region $\left(F_{(1,18)}=1.793, p>0.05\right)$ or stimulation $\left(F_{(1,18)}=2.458, p>0.05\right)$; however, we did detect a brain region $\times$ stimulation interaction $\left(F_{(1,18)}=5.046, p=0.037\right)$. Stimulation of the right rSMG, but not the Mid-Occ region, induced a large deviation in both the $\mathrm{N} 1$ and $\mathrm{CNV}$ components of the early and late measurement windows, characterized by significantly higher negative amplitudes (Fig. 4a). Similar to the comparison stimulus, negative amplitudes were larger during the CNV portion than the early N1 portion $\left(F_{(1,18)}=9.883, p=0.03\right)$, but no interaction with brain region, stimulation, and measurement window was detected $\left(F_{(1,18)}=0.023, p>0.05\right)$, indicating that the effect of rSMG stimulation was not larger for one window. A second analysis demonstrated no difference in either measurement window in non-TMS conditions between the day of stimulation (all $p>0.05)$.

\section{Correlation results}

We reasoned that, if rTMS induces a change in performance on the duration judgment task and this change is indexed by the $\mathrm{CNV}$, one would expect a correlation between the tendency to respond "longer" and the magnitude of the CNV differences between rTMS and non-rTMS trials. Consistent with this prediction, we found a significant correlation between the size of the difference in the late measurement window waveforms and the difference in behavioral performance for rSMG stimulation across subjects (Pearson's $r=0.526, p=0.021$ ) (Fig. 5a). A significant Spearman's nonparametric correlation was also found, indicating that the result was not attributable to outliers (Spearman's $\rho=0.476, p=0.04$ ). A similar analysis for Mid-Occ stimulation did not reveal a significant correlation (Pearson's $r=$ 0.016, $p>0.05$; Spearman's $\rho=-0.004, p>0.05$ ) (Fig. $5 d$ ). Furthermore, a significant correlation was not found for the early measurement window, encompassing the $\mathrm{N} 1$ waveform, and behavioral performance for either rSMG (Pearson's $r=0.273, p>$ 0.05; Spearman's $\rho=0.395, p>0.05$ ) (Fig. $5 b$ ) or Mid-Occ (Pearson's $r=0.009, p>0.05$; Spearman's $\rho=-0.125, p>0.05$ ) stimulation (Fig. $5 c$ ), indicating that the effect was specific to the late measurement window and the $\mathrm{CNV}$ waveform. Thus, the data demonstrate that, for rSMG stimulation, subjects who showed the largest increase in CNV amplitude between stimulation and no-stimulation trials also showed the largest decrease in reporting the second stimulus as longer.

We additionally calculated scalp topographies to investigate the spatial extent of rTMS-induced correlations by measuring the same correlation at other electrode sites. The results of this exploratory analysis revealed that the positive correlation between behavioral shifts and CNV amplitude resulting from rTMS was maximal at the frontocentral sites of interest, at electrode $\mathrm{Cz}$ (Fig. $5 e$ ). Furthermore, we found a negative correlation for left parietal electrodes, maximal at CP5 (Pearson's $r=-0.624, p=0.004$; Spearman's $\rho=-0.532, p=0.019$ ) (Fig. 6a), and within the early measurement window (uncorrected for multiple comparisons). Accordingly, this negative correlation demonstrated that subjects who showed little or no behavioral differences resulting from rTMS exhibited an increase in negative amplitude at these sites. Although the effect was outside our hypothesized region of interest, waveforms at this site exhibited an increase in negative amplitude during the early measurement window (Fig. 6b). However, no significant difference between TMS and no-TMS was detected during this time period $\left(t_{(18)}=\right.$ $1.642, p>0.05)$; therefore, we stress caution in the interpretation of this finding.

Because bias modulates the effect of rSMG stimulation, an effect of bias should also be present on the CNV if this waveform serves as an index of temporal encoding. We subsequently reanalyzed our EEG data to include the bias covariate; consistent with 
a

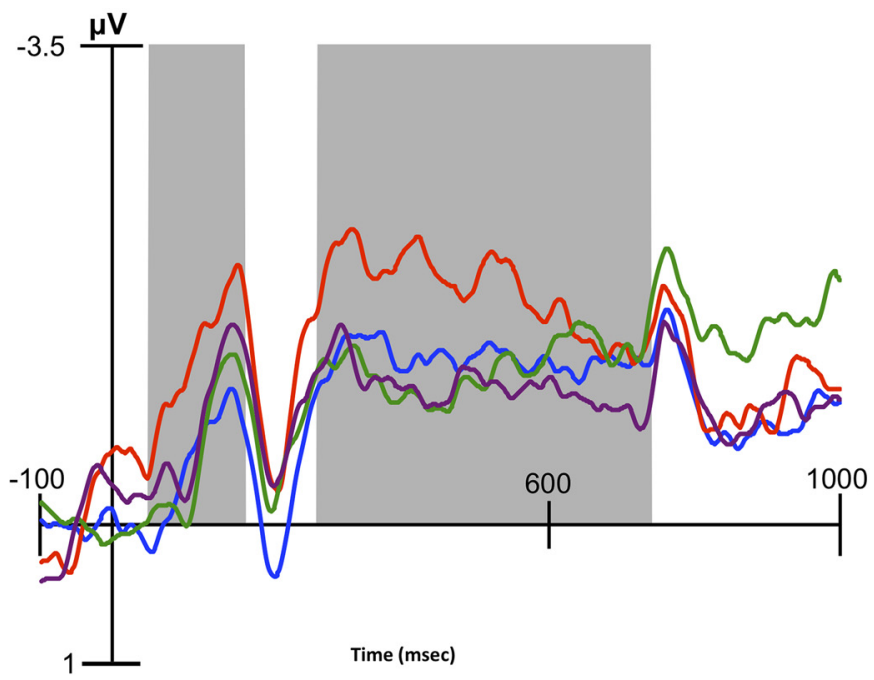

Comparison

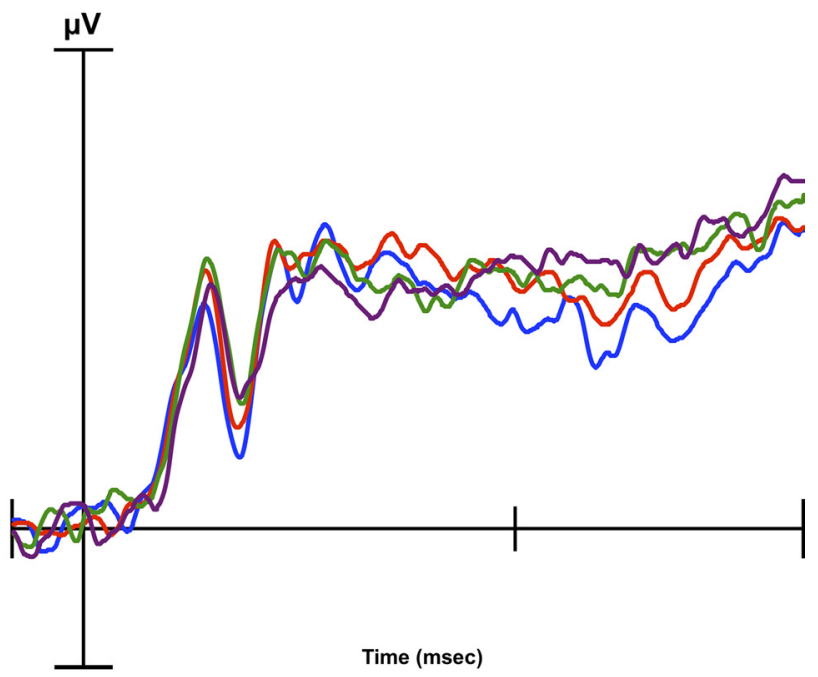

b

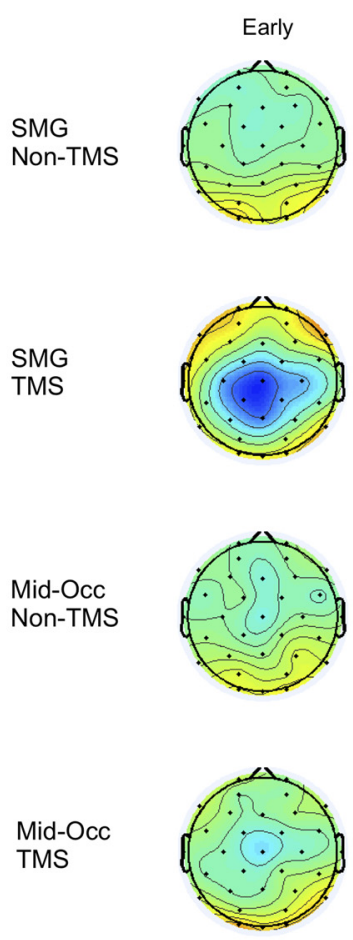

\section{Standard}

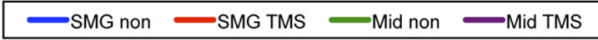

\section{Standard}
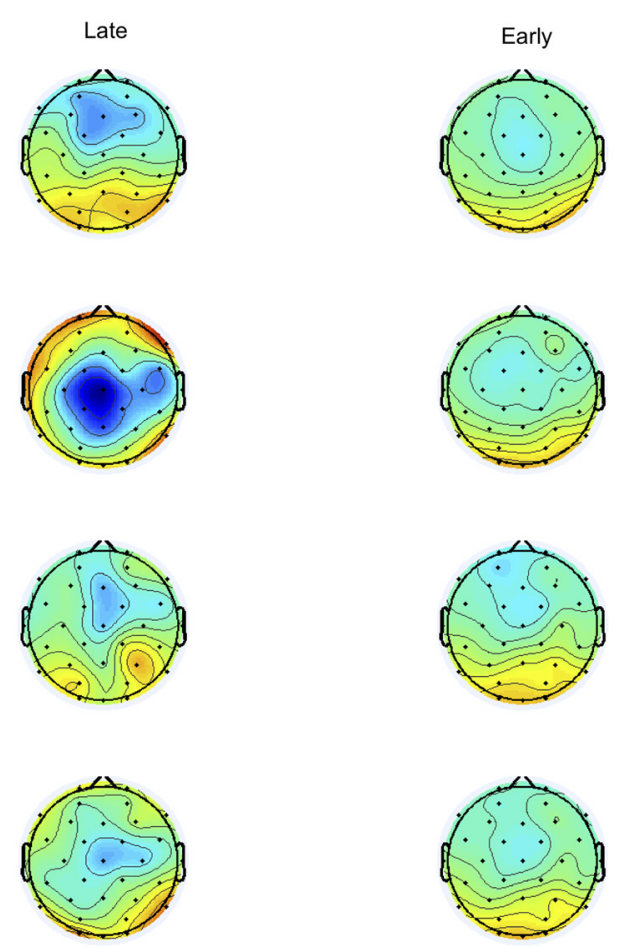

\section{Comparison}

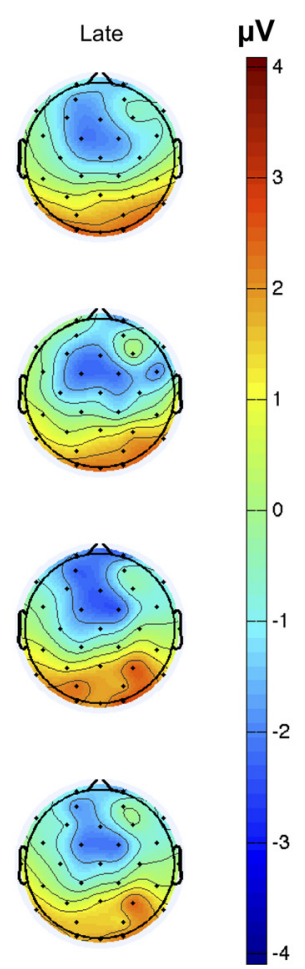

Figure 4. a, Grand-averaged waveforms for all subjects, averaged across frontocentral scalp recording sites for standard and comparison stimulus presentations in rTMS and non-rTMS conditions for rSMG and Mid-0cc-parietal junction (Mid) stimulation sites. Shaded areas represent the measurement windows derived from global estimates and encompass early and late processing components. rSMG stimulation trials demonstrated a significantly larger negative amplitude N1 and CNV during standard stimulus presentation; no changes were detected for comparison stimulus presentation in either condition. Comparison stimulus waveforms are depicted for the average of all three comparison durations. Negativity is plotted upward. $\boldsymbol{b}$, Scalp topographies for both the early and late measurement windows for both stimulation conditions. Negativity was maximal at frontocentral sites.

our behavioral analysis, we found a three-way interaction between brain region, stimulation, and bias $\left(F_{(1,17)}=5.317, p=\right.$ $0.034)$ during the standard interval encoding. Specifically, we found a significant positive correlation (Pearson's $r=0.447, p=$ 0.055 ; Spearman's $\rho=0.536, p=0.018$ ) between our bias covariate and the difference in amplitude for rSMG stimulation in the late measurement window; larger inherent bias to judge the second stimulus as shorter was associated with a smaller difference in CNV amplitude between stimulation conditions. No significant effects were found for the early measurement window, variance measures, or for either window after Mid-Occ stimulation (all $p>0.05)$. 


\section{Discussion}

Stimulation of the rSMG $500 \mathrm{~ms}$ before the onset of the standard visual stimulus in a temporal discrimination task reduced the proportion of trials on which the comparison was judged to be longer. This pattern of performance suggests that rTMS causes the subsequently encoded interval to be perceived as longer (Wiener et al., 2010a). Additional analyses demonstrate that the effect is not attributable to confounds such as response bias and click-train intensity. Grand-averaged waveforms at frontocentral recording sites during the presentation of both the standard and comparison stimuli were characterized by two negative peaks: one occurring early after stimulus onset and peaking at $\sim 155 \mathrm{~ms}$ and a second sustained negative response occurring at 280 ms. These two time windows correspond, respectively, to the visual $\mathrm{N} 1$ and $\mathrm{CNV}$ components (Brunia et al., 2011; Pratt, 2011). Crucially, rTMS to the rSMG induced significantly larger negative amplitudes in both the early (N1) and late (CNV) measurement windows. Moreover, the size of the increase in $\mathrm{CNV}$, but not N1, amplitude correlated with the size of the reduction in judging the second stimulus as longer. Although previous investigations (Macar et al., 1999) have noted a correlation between CNV amplitude and perceived duration length, this finding has been difficult to replicate (Kononowicz and van Rijn, 2011). This difficulty may stem from the use of the $\mathrm{CNV}$ amplitude as an absolute measure; because of substantial variability in $\mathrm{CNV}$ amplitude (Kropp et al., 2000), we contrasted the CNV amplitude across different conditions, thereby enhancing sensitivity. Additionally, we assessed CNV amplitude during the standard stimulus rather than the comparison stimulus, because measurements taken during the latter interval may be confounded by decision-making or motor preparation. Our results provide for the first time an example of rTMS-induced changes in $\mathrm{CNV}$ amplitude that correlate with duration judgments.

Our data are consistent with influential models of temporal processing (Gibbon et al., 1984; Zakay and Block, 1995) that incorporate an internal "clock" consisting of a pacemaker that generates pulses or beats, an accumulator that tracks the number of beats, as well as decision-making and memory components. In recent years, the supplementary and presupplementary motor areas (hereafter SMAs) have emerged as a strong candidate for the neural locus of the accumulator mecha-
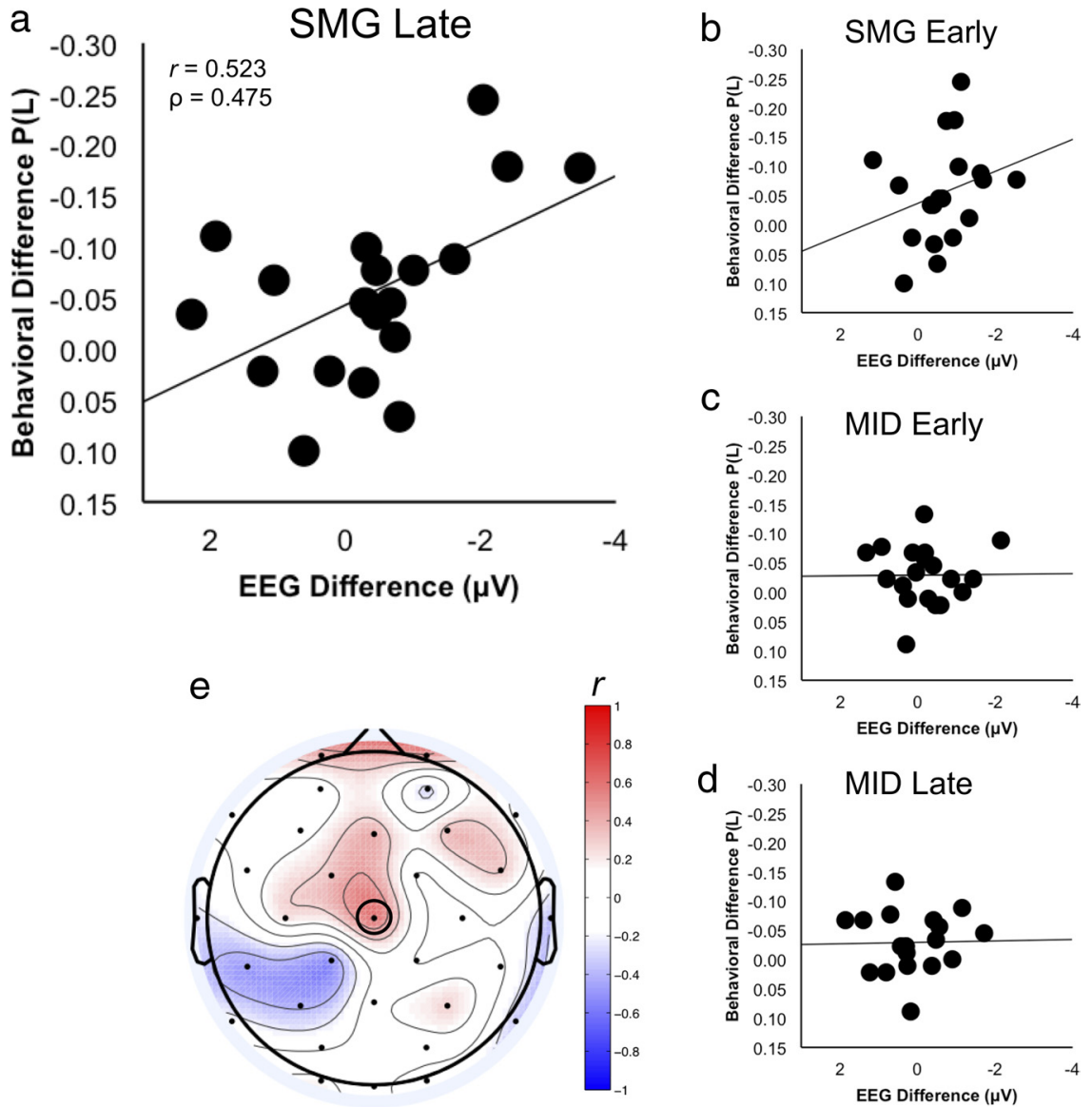

Figure 5. $\boldsymbol{a}$, Positive correlation between rSMG effect on temporal discrimination and CNV amplitude (late measurement window). The behavioral difference was calculated as the mean difference in the proportion of trials on which subjects judged the second stimulus as longer between stimulation conditions and across duration length $\left[P(L)_{\text {non-TMS }}-P(L)_{\text {TMS }}\right]$, whereas the EEG difference was the difference in mean amplitude between stimulation conditions ( $\mathrm{EEG}_{\mathrm{TMS}}-\mathrm{EEG}_{\text {non-TMS }}$ ). Pearson's and Spearman's correlation coefficients are shown for the significant correlation. Subjects who showed the largest increase in perceived duration length also showed the largest increase in CNV amplitude. $\boldsymbol{b}-\boldsymbol{d}$, No such correlation was found for N1 (early measurement window) amplitude or midline (MID) stimulation at either window. e, Scalp topographies of Pearson's correlation coefficients are also shown. The positive correlation between increases in duration length and CNV negative amplitude was maximal at electrode $\mathrm{Z}$.

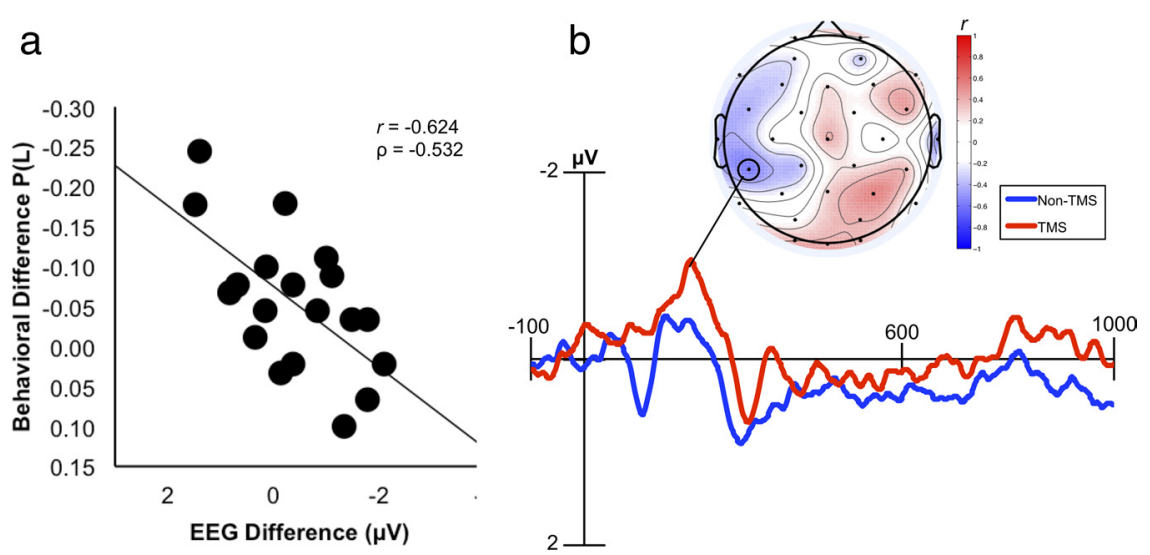

Figure 6. $\boldsymbol{a}$, Negative correlation between mean EEG amplitude and SMG behavioral effect, maximal at left parietal electrode CP5 during the early measurement window. Subjects who showed smaller shifts in behavior exhibited a larger difference in amplitude. $\boldsymbol{b}$, Grand-averaged waveforms for CP5 are displayed; despite the correlation, no significant difference between mean amplitude was detected ( $p>0.05$ ).

nism (Casini and Vidal, 2011). Several lines of evidence support this claim. First, fMRI activation within the SMAs increases monotonically during the encoding of temporal duration (Rao et al., 2001; Wencil et al., 2010). Second, BOLD activity within the 
SMAs has been found to positively covary with CNV amplitude (Nagai et al., 2004). Third, single-cell recordings in the SMAs in monkeys exhibit ramping firing-rate patterns during temporal encoding (Mita et al., 2009). Finally, the SMAs exhibit a high likelihood of activation across all explicit time perception tasks (Wiener et al., 2010b).

The finding that stimulation of the rSMG alters the CNV, a waveform generated at least in part by the SMAs, suggests that the rSMG and SMAs are closely interconnected components of the distributed neural system that mediates temporal processing. Indeed, there is both anatomic (Eickhoff et al., 2010) and physiologic (Battelli et al., 2007) evidence of interactions between the rSMG and SMA. Furthermore, neuroimaging studies using simultaneous fMRI and EEG also demonstrate that parametric variations in CNV amplitude, which activate the SMAs, also include a frontoparietal network of structures encompassing the right inferior parietal lobe (Nagai et al., 2004; Scheibe et al., 2010).

One might envision at least two mechanisms by which rSMG stimulation leads to a change in temporal processing in the SMAs. First, neurons in the rSMG may serve as the pacemaker for the internal clock; rTMS would thus increase the firing rate of the pacemaker, leading to a larger number of pulses entering the accumulator, subserved by the SMAs, and a longer judgment of duration. The resultant increase in accumulation in the SMAs would manifest as a larger-amplitude CNV. Alternatively, on the basis of studies demonstrating that temporal processing is influenced by attention (Zakay and Block, 1995), one might speculate that the rSMG serves as an attentional "gate" that modulates input to the accumulator, subserved by the SMAs.

An additional finding in the present study is that rSMG stimulation induced an increase in amplitude during the early (N1) time window. Several previous studies have noted changes in the N1 response during temporal discrimination and attention tasks (Bendixen et al., 2005; Correa and Nobre, 2008; Chen et al., 2010). These studies demonstrate that the $\mathrm{N} 1$ effect is enhanced when subjects attend to temporal features or judge stimuli to be longer. Consistent with this finding, the visual N1 component has commonly been associated with attentional orienting (Vogel and Luck, 2000). In the present study, the $\mathrm{N} 1$ effect at frontocentral electrodes did not correlate with duration judgments. This suggests that the enhancement of the early $\mathrm{N} 1$ reflects a general increase in the attentional allocation to time, whereas the late $\mathrm{CNV}$ reflects the accumulation of temporal information toward an estimate of duration. This finding is consistent with the suggestion that attention to time and temporal accumulation are separate processes, with attention influencing the subsequent level of accumulation. In conjunction with the effect on CNV amplitude, we suggest that the combination of N1 and CNV effects implicates the rSMG in the attentional allocation of resources to time and that this mechanism is accomplished by functional connectivity between this region and the SMAs. Consistent with this notion, parietal activity commonly precedes frontal activation when directing attention (Green and MacDonald, 2008) and prefrontal-parietal coactivations, including the SMAs, are essential for sustaining attention (Ojeda et al., 2002).

The dissociation between early and late effects also demonstrated a differential effect of the early response window at a region outside the frontocentral cluster of interest. We found a negative correlation with duration judgments; larger amplitudes at CP5 after rTMS were associated with less of a behavioral effect. One possibility, then, is that variability in the EEG response to rTMS in the present study represents individual differences in strategy. Because the left hemisphere has been associated with implicit temporal attention (Coull and Nobre, 2008; Wiener et al., 2010c), differences at this site may reflect changes in attentional orienting to temporal features. However, we note there were no significant differences in the mean amplitude between conditions at this site, and so this effect must be interpreted with caution.

A second novel finding of the present study is the modulation of behavioral and EEG results by bias, as derived from the baseline psychometric task. Decision bias in time perception may take the form of positive or negative time-order errors, in which the subject displays an inherent predilection to judge the comparison stimuli as shorter or longer, respectively (Hellström, 1985). Most subjects in our study displayed some form of bias, with the majority of subjects exhibiting a positive time-order error, indicating a preference to judge the comparison stimulus as shorter across all interval variations. We found that subjects who were strongly biased to judge the second stimulus as shorter do not show as large an effect as those with less bias. We note that one possibility for this difference is that subjects with greater inherent bias rely less on sensory evidence in generating temporal estimates (Jazayeri and Shadlen, 2010). Therefore, parietal stimulation may have little effect in those subjects if rTMS led to a disruption of sensory encoding.

Additionally, EEG analyses demonstrated an effect of bias. We found that, for the $\mathrm{CNV}$, but not N1, larger positive bias scores were associated with smaller differences induced by rSMG stimulation. This finding is also consistent with the hypothesis that the CNV serves as an index of temporal encoding. Previous studies have demonstrated that the CNV signal covaries with the probability of an upcoming decision (Scheibe et al., 2010), suggesting that the CNV is influenced by previous expectations. Bias to judge the comparison stimulus as short is thus likely reflected in the CNV signal if it represents an index of the temporal accumulation. Two hypothetical neural mechanisms for the influence of bias have been suggested, with bias influencing either the starting point of accumulation or the drift rate to a threshold boundary (Diederich and Busemeyer, 2006). Because the influence of bias on the starting point would occur early in stimulus encoding and we found no effect of bias on N1, this seems an unlikely account for our data. Our data are more consistent with the notion of a change in drift rate, as hypothesized recently by Simen et al. (2011).

\section{Conclusions}

We demonstrate that rTMS-induced alterations in CNV amplitude index the state of temporal encoding, with larger amplitudes associated with longer perceived durations. These results are independent of changes in decision-making, bias, click trains, and motor preparation and occur at the late stage of temporal encoding rather than the initial registration of a visual event. We propose that temporal encoding of subsecond intervals entails the attending of sensory information by association cortices. By virtue of connections between these cortices and the SMAs, this input is integrated to generate a representation of duration, which can be indexed by changes in the CNV.

\section{References}

Battelli L, Pascual-Leone A, Cavanagh P (2007) The "when" pathway of the right parietal lobe. Trends Cogn Sci 11:204-210. 
Bendixen A, Grimm S, Schröger E (2005) Human auditory event-related potential predict duration judgments. Neurosci Lett 383:284-288.

Berardelli A, Inghilleri M, Rothwell JC, Romeo S, Curr à A, Gilio F, Modugno N, Manfredi M (1998) Facilitation of muscle evoked responses after repetitive cortical stimulation in man. Exp Brain Res 122:79-84.

Brainard DH (1997) The psychophysics toolbox. Spat Vis 10:433-436.

Brunia CH, van Boxtel GJ, Bocker KB (2011) Negative slow waves as indices of anticipation: the Bereitschaftspotential, the contingent negative variation, and the stimulus-preceding negativity. In: The Oxford handbook of event-related potential components (Luck SA, Kappenman E, eds). Oxford, UK: Oxford UP.

Buhusi CV, Meck WH (2005) What makes us tick? Functional and neural mechanisms of interval timing. Nat Rev Neurosci 6:755-765.

Burle B, Casini L (2001) Dissociation between activation and attention effects in time estimation: implications for internal clock models. J Exp Psychol Hum Percept Perform 27:195-205.

Casini L, Vidal F (2011) The SMAs: neural substrate of the temporal accumulator? Front Integr Neurosci 5:35.

Chen Y, Huang X, Yang B, Jackson T, Peng C, Yuan H, Liu C (2010) An event-related potential study of temporal information encoding and decision making. Neuroreport 21:152-155.

Cicchini GM, Arrighi R, Cecchetti L, Giusti M, Burr DC (2012) Optimal encoding of interval timing in expert percussionists. J Neurosci 32:1056-1060.

Correa A, Nobre AC (2008) Neural modulation by regularity and passage of time. J Neurophysiol 100:1649-1655.

Coull J, Nobre A (2008) Dissociating explicit timing from temporal expectation with fMRI. Curr Opin Neurobiol 18:137-144.

Diederich A, Busemeyer JR (2006) Modeling the effects of payoff on response bias in a perceptual discrimination task: bound-change, drift-ratechange, or two-stage-processing hypothesis. Percept Psychophys 68: 194-207.

Eickhoff SB, Jbabdi S, Caspers S, Laird AR, Fox PT, Zilles K, Behrens TE (2010) Anatomical and functional connectivity of cytoarchitectonic areas within the human parietal operculum. J Neurosci 30:6409-6421.

Fan J, Kolster R, Ghajar J, Suh M, Knight RT, Sarkar R, McCandliss BD (2007) Response anticipation and response conflict: an event-related potential and functional magnetic resonance imaging study. J Neurosci 27:2272-2282.

Gibbon J, Church RM, Meck WH (1984) Scalar timing in memory. Ann NY Acad Sci 423:52-77.

Gontier E, Le Dantec C, Leleu A, Paul I, Charvin H, Bernard C, Lalonde R, Reba ï M (2007) Frontal and parietal ERPs associated with duration discriminations with or without task interference. Brain Res 1170:79-89.

Green JJ, MacDonald JJ (2008) Electrical neuroimaging reveals timing of attentional control in human brain. PLoS Biol 6:730-738.

Hamidi M, Slagter HA, Tononi G, Postle BR (2010) Brain responses evoked by high-frequency repetitive transcranial magnetic stimulation: an eventrelated potential study. Brain Stimul 3:2-14.

Hellström A (1985) The time-order error and its relatives: mirrors of cognitive processes in comparing. Psych Bull 97:35-61.

Ikeda A, Shibasaki H, Kaji R, Terada K, Nagamine T, Honda M, Kimura J (1997) Dissociation between contingent negative variation (CNV) and Bereitschaftspotential (BP) in patients with parkinsonism. Electroencephalogr Clin Neurophysiol 102:142-151.

Iwahashi M, Katayama Y, Ueno S, Iramina K (2009) Effect of transcranial magnetic stimulation on P300 of event-related potential. Conf Proc IEEE Eng Med Biol Soc 2009:1359-1362.

Jazayeri M, Shadlen MN (2010) Temporal context calibrates interval timing. Nat Neurosci 13:1020-1026.

Jung TP, Makeig S, Westerfield M, Townsend J, Courchesne E, Sejnowski TJ (2000) Removal of eye activity artifacts from visual event-related potentials in normal and clinical subjects. Clin Neurophysiol 111:1745-1758.

Koessler L, Maillard L, Benhadid A, Vignal JP, Felblinger J, Vespignani H, Braun M (2009) Automated cortical projection of EEG sensors: anatomical correlation via the international $10-10$ system. Neuroimage 46:64-72.

Kononowicz TW, van Rijn H (2011) Slow potentials in time estimation: the role of temporal accumulation and habituation. Front Integr Neurosci $5: 48$.

Korhonen RJ, Hernandez-Pavon JC, Metsomaa J, Mäki H, Ilmoniemi RJ, Sarvas J (2011) Removal of large muscle artifacts from transcranial mag- netic stimulation-evoked EEG by independent component analysis. Med Biol Eng Comput 49:397-407.

Kropp P, Kiewitt A, Göbel H, Vetter P, Gerber WD (2000) Reliability and stability of contingent negative variation. Appl Psychophysiol Biofeedback 25:33-41.

Le Dantec C, Gontier E, Paul I, Charvin H, Bernard C, Lalonde R, Rebai M (2007) ERPs associated with visual duration discriminations in prefrontal and parietal cortex. Acta Psychol (Amst) 125:85-98.

Lehmann D, Skrandies W (1980) Reference-free identification of components of checkerboard-evoked multichannel potential fields. Electroencephalogr Clin Neurophysiol 48:609-621.

Levit-Binnun N, Litvak V, Pratt H, Moses E, Zaroor M, Peled A (2010) Differences in TMS-evoked responses between schizophrenia patients and healthy controls can be observed without a dedicated EEG system. Clin Neurophysiol 121:332-339.

Lieberman HR, Pentland AP (1983) Microcomputer-based estimation of psychophysical thresholds: the best PEST. Behav Res Methods 14:21-25.

Macar F, Vidal F (2003) The CNV peak: an index of decision making and temporal memory. Psychophysiology 40:950-954.

Macar F, Vidal F (2004) Event-related potentials as indices of time processing: a review. J Psychophysiol 18:89-104.

Macar F, Vidal F, Casini L (1999) The supplementary motor area in motor and sensory timing: evidence from slow brain potentials. Exp Brain Res 125:271-280.

Mita A, Mushiake H, Shima K, Matsuzaka Y, Tanji J (2009) Interval time coding by neurons in the presupplementary and supplementary motor areas. Nat Neurosci 12:502-507.

Murray MM, Brunet D, Michel CM (2008) Topographic ERP analyses: a step-by-step tutorial review. Brain Topogr 20:249-264.

Nagai Y, Critchley HD, Featherstone E, Fenwick PB, Trimble MR, Dolan RJ (2004) Brain activity relating to the contingent negative variation: an fMRI investigation. Neuroimage 21:1232-1241.

Ng KK, Tobin S, Penney TB (2011) Temporal accumulation and decision processes in the duration bisection task revealed by contingent negative variation. Front Integr Neurosci 5:77.

Ojeda N, Ortuño F, Arbizu J, López P, Martí-Climent JM, Peñuelas I, Cervera-Enguix S (2002) Functional neuroanatomy of sustained attention in schizophrenia: contribution of parietal cortices. Hum Brain Mapp 17:116-130.

Pentland A (1980) Maximum likelihood estimation: the best PEST. Percept Psychophys 28:377-379.

Penton-Voak IS, Edwards H, Percival A, Wearden JH (1996) Speeding up an internal clock in humans? Effects of click trains on subjective duration. J Exp Psychol Hum Percept Perform 22:307-320.

Pflieger ME (2001) Theory of a spatial filter for removing ocular artifacts with preservation of EEG. EMSE Workshop, Princeton, September 7-8.

Praamstra P, Kourtis D, Kwok HF, Oostenveld R (2006) Neurophysiology of implicit timing in serial choice reaction-time performance. J Neurosci 26:5448-5455.

Pratt H (2011) Sensory ERP components. In: The Oxford handbook of event-related potential components (Luck SA, Kappenman E, eds). Oxford, UK: Oxford UP.

Rao SM, Mayer AR, Harrington DL (2001) The evolution of brain activation during temporal processing. Nat Neurosci 4:317-323.

Scheibe C, Ullsperger M, Sommer W, Heekeren HR (2010) Effects of parametrical and trial-to-trial variation in prior probability processing revealed by simultaneous electroencephalogram/functional magnetic resonance imaging. J Neurosci 30:16709-16717.

Simen P, Balci F, de Souza L, Cohen JD, Holmes P (2011) A model of interval timing by neural integration. J Neurosci 31:9238-9253.

Tarantino V, Ehlis AC, Baehne C, Boreatti-Huemmer A, Jacob C, Bisiacchi P, Fallgatter AJ (2010) The time course of temporal discrimination: an ERP study. Clin Neurophysiol 121:43-52.

van Rijn H, Kononowicz TW, Meck WH, Ng KK, Penney TB (2011) Contingent negative variation and its relation to time estimation: a theoretical evaluation. Front Integr Neurosci 5:91.

Veniero D, Bortoletto M, Miniussi C (2009) TMS-EEG co-registration: on TMS-induced artifact. Clin Neurophysiol 120:1392-1399.

Vogel EK, Luck SJ (2000) The visual N1 component as an index of a discrimination process. Psychophysiology 37:190-203.

Walsh V, Pascual-Leone A (2003) Transcranial magnetic stimulation: a 
neurochronometrics of mind. Cambridge, MA: Massachusetts Institute of Technology.

Walter WG, Cooper R, Aldridge VJ, McCallum WC, Winer AL (1964) Contingent negative variation: an electric sign of sensorimotor association and expectancy in the human brain. Nature 25:380-384.

Wencil EB, Coslett HB, Aguirre GK, Chatterjee A (2010) Carving the clock at its component joints: neural bases for interval timing. J Neurophysiol 104:160-168.

Wichmann FA, Hill NJ (2001a) The psychometric function. I. Fitting, sampling, and goodness of fit. Percept Psychophys 63:1293-1313.

Wichmann FA, Hill NJ (2001b) The psychometric function. II. Bootstrapbased confidence intervals and sampling. Percept Psychophys 63:13141329.
Wiener M, Hamilton R, Turkeltaub P, Matell MS, Coslett HB (2010a) Fast forward: rSMG stimulation alters time measurement. J Cogn Neurosci 22:23-31.

Wiener M, Turkeltaub P, Coslett HB (2010b) The image of time: a voxelwise meta-analysis. Neuroimage 49:1728-1740.

Wiener M, Turkeltaub PE, Coslett HB (2010c) Implicit timing activates the left inferior parietal cortex. Neuropsychologia 48:3967-3971.

Xuan B, Chen XC, He S, Zhang DR (2009) Numerical magnitude modulates temporal comparison: an ERP study. Brain Res 1269:135-142.

Zakay D, Block RA (1995) An attentional gate model of prospective time estimation. In: Time and the dynamic control of behavior (Richelle M, De Keyser V, D’Ydewalle G, Vandierendonck A, eds), pp 167-178. Liege, Belgium: Université de Liege. 